\title{
A Western blot approach to detection of human plasma protein conjugates derived from D-penicillamine
}

\author{
C A Laycock, M J I Phelan, R C Bucknall, J W Coleman
}

\begin{abstract}
Objectives-To develop and apply an immunochemical approach to the study of drug-plasma protein conjugates derived from the anti-arthritic drug D-penicillamine (DP).

Methods-An antiserum with specificity for protein-conjugated DP was raised in a rabbit. Plasma samples from patients receiving DP or from incubations of isolated normal plasma with DP were analysed for DP-derived conjugates by Western blotting using the anti-drug antibody.

Results-A single DP-positive protein band was detected in plasma samples from $15 / 16$ patients with rheumatoid arthritis receiving DP but in none of 20 patients of similar disease status who had not taken DP. The positive band appeared in patients' plasma during the course of treatment with DP. It was seen under nonreducing but not reducing conditions indicating that the drug is disulphide linked to the protein. The drug-modified protein migrated to a position intermediate between the trailing edge of albumin and the leading edge of transferrin (both non-reduced) suggesting a molecular weight of between 66 and 77 kDa. Incubations of normal human plasma, but not purified albumin or transferrin, with low concentrations of DP generated the same distinct band plus several less intense DP-positive bands.

Conclusions-Drug-plasma protein conjugates derived from DP in vivo and in vitro can be detected immunochemically by the Western blot method. Theories of DP immunotoxicity have implicated antigenicity of the drug, but this is the first immunochemical demonstration of a potential DP-derived antigen in human plasma. The method we describe may have application to studies of the relationship between DP antigenicity and toxicity.
\end{abstract}

(Ann Rheum Dis 1994; 53: 256-260)

D-penicillamine (DP) is a therapeutic agent in current use as a second line therapy for rheumatoid arthritis, and, by virtue of its metal-chelating and disulphide-reducing properties as a mainstay treatment for Wilson's disease, lead poisoning and cystinuria. The benefits of the drug in rheumatoid arthritis are severely curtailed by a high incidence of adverse reactions which necessitate withdrawal in up to $40 \%$ of patients. ${ }^{12}$ Common side effects include skin rashes, fever and proteinuria. More rarely but more seriously, agranulocytosis, thrombocytopenia, and autoimmune disorders such as myasthenia gravis and systemic lupus erythematosus have been reported. ${ }^{12}$ The nature of these side effects, and their reported association with HLA DR $3^{3}$ has led to suggestions that adverse reactions to DP are mediated by the immune system, ${ }^{2-6}$ and the capacity of DP to conjugate by disulphide linkage to plasma proteins ${ }^{78}$ and to cell surfaces $^{9}$ may confer antigenic (haptenic) potential upon the molecule. Reports of antibody responses in patients ${ }^{6}{ }^{10}$ and $T$ cell responses in mice ${ }^{11} 12$ directed against DPderived determinants support the notion that DP can act as an immunologically operative hapten.

Studies of the disposition of DP in vivo employing either $\left[{ }^{14} \mathrm{C}\right]$-labelled DP in rats $^{78}$ or chemical extraction and HPLC analysis in humans ${ }^{13} 14$ provide useful information on drug-protein conjugation but this may not necessarily reflect generation of a functionally relevant hapten, that is, a DP-derived antigenic determinant that can be recognised by antibodies and/or lymphocytes. In this study therefore we sought to develop an immunochemical method for detection of DPderived conjugates. This approach may have application to detection of the haptenic derivative in plasma samples obtained from patients receiving the drug. Identification of drug-derived conjugates may be an important first step in understanding the processes of DP immunogenicity and antigenicity and may throw light on mechanisms of DP immunotoxicity.

\section{Patients and methods}

PATIENTS AND PLASMA SAMPLES

Plasma was obtained under sterile conditions from heparinised venous blood obtained from the following groups of patients and healthy volunteers: 16 patients with rheumatoid arthritis (ARA criteria) who had been receiving D-penicillamine (DP) for at least three months; 10 patients with rheumatoid arthritis who started treatment with DP during the course of the study; 10 patients with rheuma- 
toid arthritis who received alternative second line therapy to DP (sodium aurothiomalate or methotrexate) during the course of the study; 10 healthy volunteers from laboratory staff. The daily dose of DP for patients receiving this drug was 250-750 mg. All patients with rheumatoid arthritis were also taking nonsteroidal anti-inflammatory agents during the study period.

INCUBATIONS OF NORMAL PLASMA OR PURIFIED PROTEINS WITH DP IN VITRO

Plasma was obtained from healthy volunteers who were not receiving any medication. Plasma, diluted $1: 3$ in $0.15 \mathrm{M}$ phosphatebuffered saline, $\mathrm{pH} 7 \cdot 2$ (PBS), transferrin, $2 \cdot 5$ $\mathrm{mg} / \mathrm{ml}$ in PBS (Calbiochem, Nottingham, UK), or human serum albumin, $40 \mathrm{mg} / \mathrm{ml}$ in PBS (Calbiochem), were incubated for 20 hours at $37^{\circ} \mathrm{C}$ with neutralised D-penicillamine-HCl (DP, Sigma, Poole, UK) in PBS under sterile conditions.

\section{SDS-PAGE AND WESTERN BLOTTING}

An antiserum with specificity for proteinconjugated DP was raised in a rabbit by injection of DP-keyhole limpet haemocyanin emulsified in Freund's complete adjuvant as described in more detail previously. ${ }^{15}$ The antiserum was used as a probe in Western blots to detect the DP hapten on electrophoretically separated and transferred plasma proteins. Plasma samples were diluted 1:19 in Laemmli electrophoresis buffer, ${ }^{16}$ with or without dithiothreitol $(3 \mathrm{mg} / \mathrm{ml})$ as a reducing reagent as required, and placed in a boiling water bath for five minutes before analysis by sodium dodecyl sulphate-polyacrylamide gel electrophoresis (SDS-PAGE). Samples were either used immediately or stored at $-20^{\circ} \mathrm{C}$. Each sample $(10 \mu \mathrm{l})$ was electrophoresed on SDS$7 \%$ polyacrylamide ${ }^{16}$ at a constant current of $30 \mathrm{~mA} /$ gel on a vertical slab gel system (Hoefer Mighty Small). Reduced prestained protein molecular weight markers (size range $26 \cdot 6-116$ $\mathrm{kDa}$, Sigma, Poole, UK) were run on every gel in the first and last lane. The separated proteins were electrophoretically transferred to nitrocellulose paper using a semi-dry blotter (Biometra). The blots were blocked for at least one hour in $2.5 \%$ casein in wash buffer $(0.9 \%$ $\mathrm{NaCl}, 0.121 \%$ Tris, $0.04 \%$ thimerosal, $\mathrm{pH}$ $7 \cdot 6)$. The blots were then incubated with one of the following antibody preparations diluted $1: 500$ or $1: 1000$ in $1 \%$ casein in wash buffer for 3 to 20 hours at room temperature on an orbital shaker: rabbit anti-DP antiserum, mouse monoclonal anti-human transferrin (Chemicon, London), mouse monoclonal antihuman albumin (Sigma), or goat anti-human albumin (Chemicon). After incubation with antibody the blots were washed once in wash buffer, once in detergent buffer $(0.5 \%$ Triton $\mathrm{X}-100,0 \cdot 1 \%$ SDS in wash buffer), and twice again with wash buffer, and then incubated with either horse radish peroxidase (HRPO)labelled goat anti-rabbit IgG (TAGO, Burlingame, CA, USA), HRPO-goat anti- mouse IgG (Calbiochem) or HRPO-rabbit anti-goat IgG (Calbiochem) as appropriate. HRPO-labelled antibody preparations were all used at a concentration of $1: 500$ or $1: 1000$ in wash buffer. Finally, the blots were washed four times in wash buffer, four times in Trissaline $(1 \cdot 169 \% \mathrm{NaCl}, 0.6 \%$ Tris $)$ and developed in Tris saline substrate solution containing $0.05 \%$ 4-chloro-1-naphthol (dissolved initially in methanol, final concentration $17 \%$ ) and $0 \cdot 133 \% \mathrm{H}_{2} \mathrm{O}_{2}$.

In addition to immunostaining, replicate blots of all plasma and protein samples were stained for total protein in amido black solution $(0.1 \%$ in $25 \% \mathrm{v} / \mathrm{v}$ isopropanol, $10 \%$ $\mathrm{v} / \mathrm{v}$ acetic acid) and excess stain removed in destaining solution containing $70 \%$ methanol and $2 \%$ glacial acetic in water.

\section{Results}

Immunoblots of plasma samples obtained from patients receiving treatment with DP and probed with anti-DP antibody revealed a single distinct band (fig 1). This band was not seen in samples obtained from patients with rheumatoid arthritis who were not receiving the drug (fig 1). This distinct DP-positive band was detected in plasma samples from 15/16 patients with rheumatoid arthritis who had started treatment with $D P$, in $0 / 20$ patients with similar disease status but who had not received DP, and in $0 / 10$ healthy volunteers who were receiving no medication.

When plasma samples were obtained from patients immediately before starting treatment with DP and then at monthly intervals during the treatment period, the DP-positive band was distinct at the first one-month time point and was retained at an equal intensity thereafter (fig 2).

The single distinct DP-positive protein band, that was seen in plasma samples from patients taking the drug (fig 1,2 ), was not seen when blots were developed with control normal rabbit serum in place of the specific rabbit anti-DP antiserum (data not shown). The various high molecular weight, lightly staining bands that were seen in some blots developed with anti-DP (as in fig 1) also stained with normal rabbit serum and therefore represent non-specific binding of rabbit serum immunoglobulin or components of the HRPOlabelled second antibody to the separated human plasma proteins.

The DP-positive band was seen only when plasma samples were run under non-reducing conditions, that is, in the absence of dithiothreitol, indicating that the drug was bound to the protein by disulphide linkage. The DP band in non-reduced samples migrated to a position intermediate between the reduced molecular weight markers of 48.5 and $58 \mathrm{kDa}$ (fig 1-4). It should be noted, however, that by necessity the protein molecular weight markers were run under reducing conditions to allow their resolution, and since reduced proteins show quite different electrophoretic mobilities to non-reduced proteins a direct comparison of the mobility of 


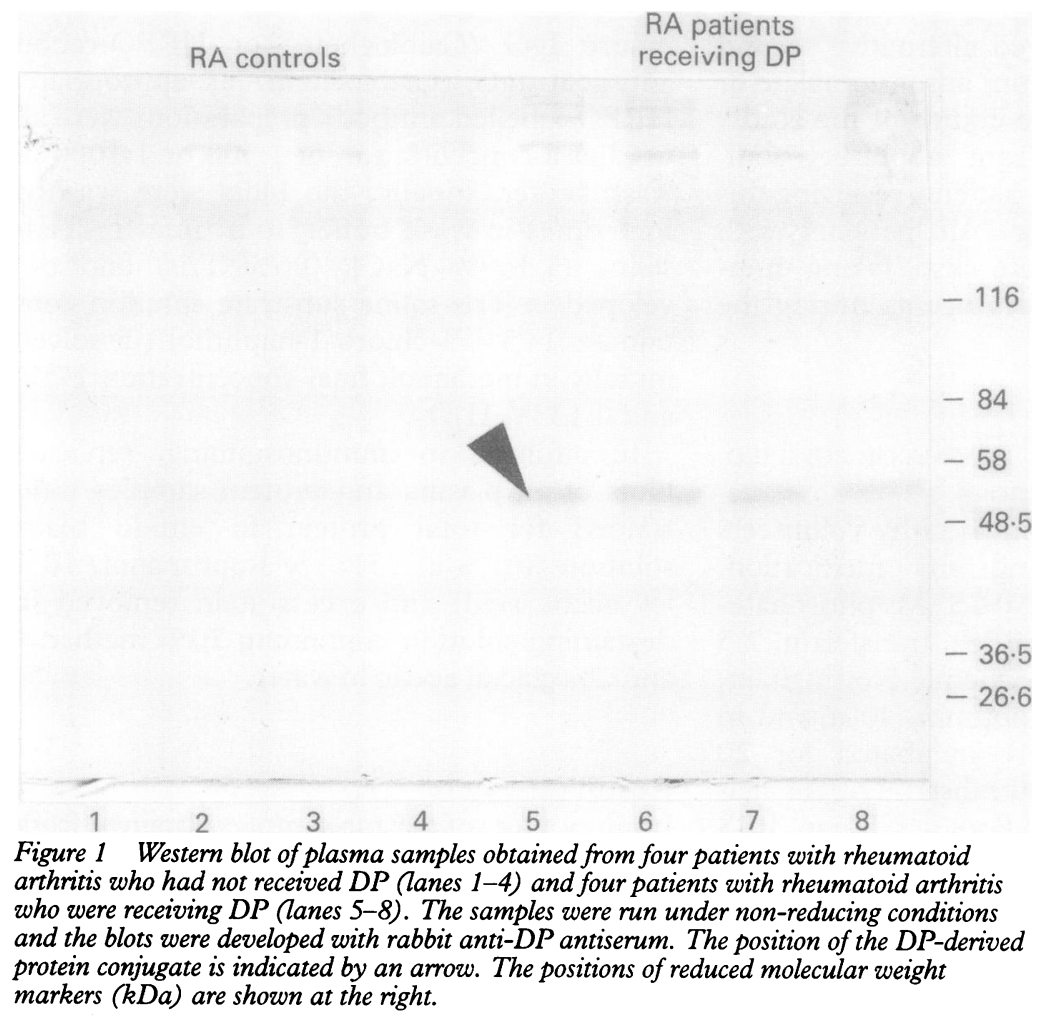
protein conjugate is indicated by an arrow. The positions of reduced molecular weigh band consistently migrated to a position that was intermediate between the albumin and transferrin bands, but was nevertheless very close to the leading edge of the transferrin (mol wt $77 \mathrm{kDa}$ ) and to the trailing edge of the albumin (mol wt $66 \mathrm{kDa}$ ). We thus tentatively assign a molecular weight to the DP-positive band of $66-77 \mathrm{kDa}$. Although close to the trailing edge of the albumin band the DP band was quite distinct from albumin since it appeared as a relatively narrow, horizontal band that was not stained by the anti-albumin antibodies, whereas the albumin band appeared as a broad band (also seen by amido black staining) with a down-curved trailing edge (fig 3 ).

In vitro incubations of DP with normal plasma yielded a concentration-dependent increase in DP binding to proteins both quantitatively and qualitatively. At low concentrations of DP $(0.06-0.13 \mathrm{mM})$ binding was detected only to a single protein band (fig 4), migrating to a position corresponding to that seen for the DP band seen in the patients' plasma samples (compare fig 4 with figs $1-3$ ). At higher concentrations of DP $(0.5 \mathrm{mM}$ and above) the above-mentioned DP band continued to increase in intensity, while a further two intensely staining DP-positive bands appeared, one at a position approximately half way between the $58 \mathrm{kDa}$ and the $84 \mathrm{kDa}$ reduced markers and one above the $116 \mathrm{kDa}$ reduced marker (fig 4). Each of these three distinct DP-induced bands and a number of other relatively weakly staining DP-induced bands (fig 4) were seen under non-reducing but not reducing conditions, again indicating that the DP-derived determinants are disulphide conjugated to their protein targets. Similar in vitro incubations of purified transferrin or albumin with comparable concentrations of DP to those shown in fig 4 led to no detectable staining with the anti-DP antibody, thus showing that DP does not bind so readily to purified albumin or transferrin as to whole plasma.

\section{Discussion}

We report that the anti-arthritic drug D-penicillamine (DP) generates a conjugate in vivo which is seen as a single protein band in Western blots of plasma samples taken from patients receiving the drug. This finding is consistent with theories, ${ }^{4-6}$ 8-12 that relate immunotoxic reactions to this drug to its potential to act as an immunologically functional hapten. The conjugate is clearly drug-specific since it was detected by immunoblot analysis with a specific, well characterised, rabbit anti-DP antiserum ${ }^{15}$ but not with normal rabbit serum as control. It is also clearly drug-induced in vivo since it was seen in plasma samples from $15 / 16$ patients with rheumatoid arthritis who were taking the drug but in none of 20 patients with rheumatoid arthritis taking other medicines or before treatment with DP. When plasma samples were taken from patients before and during treatment with DP, the conjugate was absent before treatment, but after starting sections that were either stained with rabb anti-DP, monoclonal or polyclonal antialbumin, or monoclonal anti-transferrin as appropriate. The stained blots were then realigned to their positions on the original gel. Typical results are shown in fig 3. The DP 


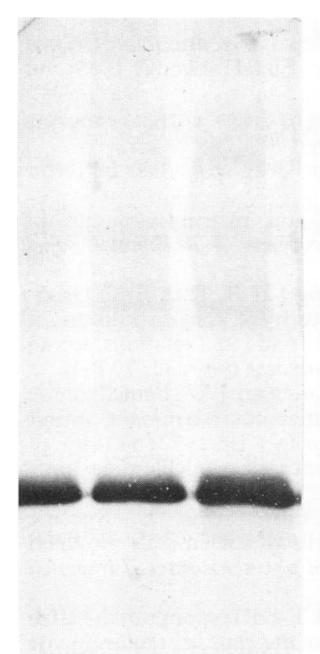

Anti-transferrin

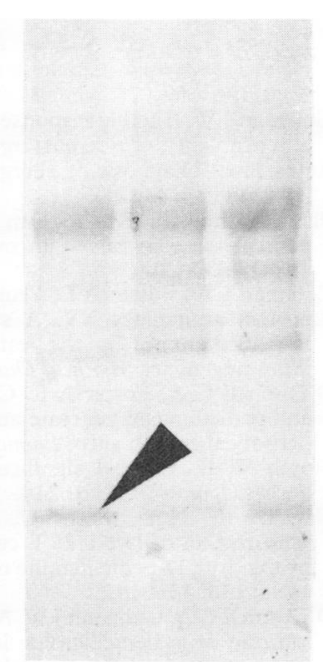

Anti-DP

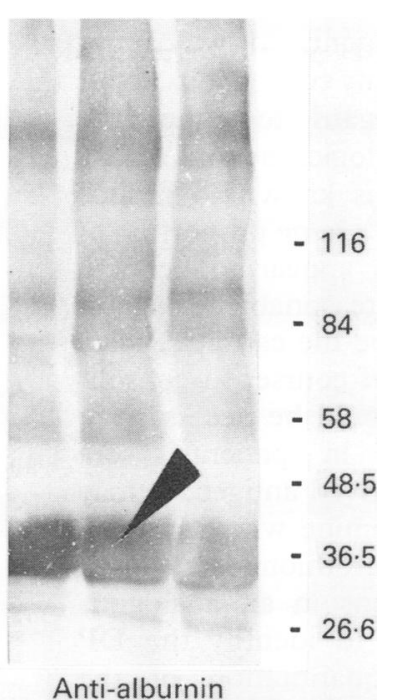

Anti-alburnin

Figure 3 Western blots of plasma samples obtained from three patients (three lanes in each panel) during courses of treatment with DP. The samples were run under non-reducing conditions and blots were developed with anti-transferrin (left hand panel), anti-DP (centre panel) or anti-albumin (right hand panel). The position of the DP band (centre panel) and albumin band (right hand panel) are indicated by arrows. The positions of reduced molecular weight markers $(k D a)$ are shown at the right.

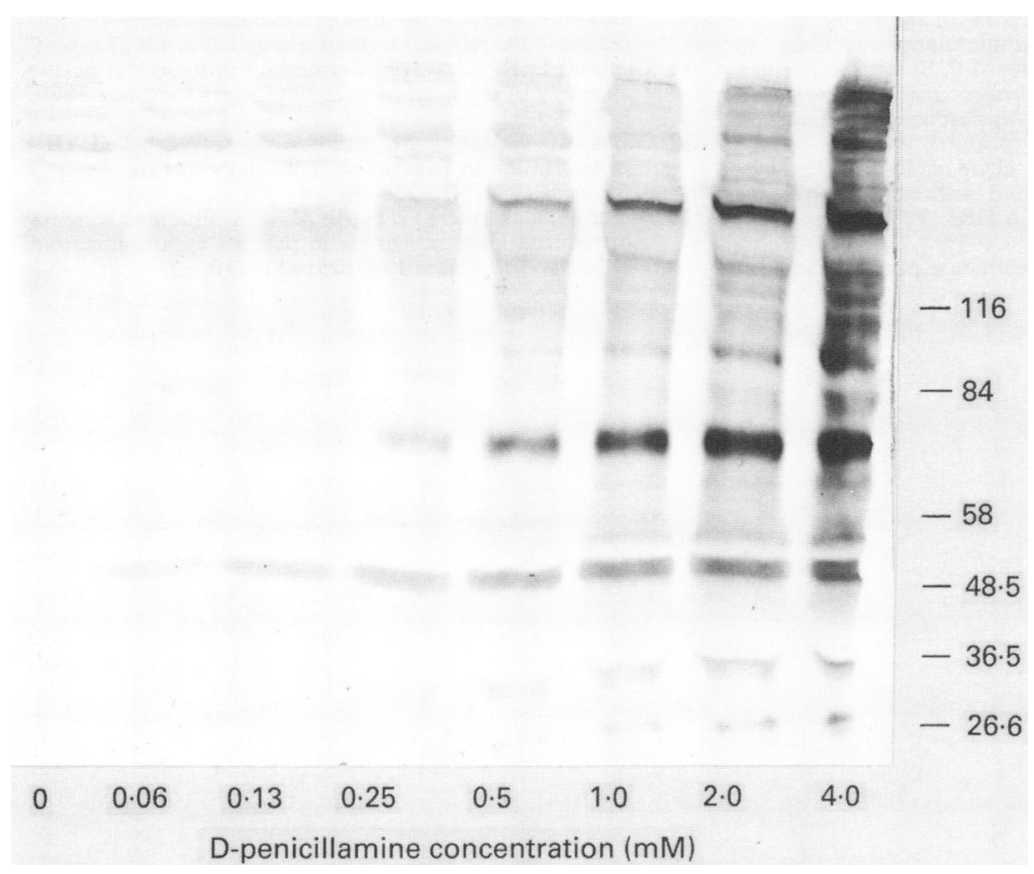

Figure 4 Western blot showing concentration-dependent generation of the DP-derived protein conjugate after incubation of normal human plasma with a range of concentrations of the drug in vitro. The samples were electrophoresed and blotted as in fig 1. The positions of reduced molecular weight markers $(k D a)$ are shown at the right.

treatment was seen clearly and persisted during the treatment period. Within the present study we were unable to monitor the disappearance of the conjugate on cessation of DP treatment, although it would certainly be of interest to do so. It should be noted also that the Western blot method does not detect all DP-protein interactions, but only those that result in generation of a conjugate accessible to antibody.

We are unable to identify the protein target for the DP conjugate generated in vivo but on the basis of its electrophoretic mobility in relation to non-reduced albumin and transferrin we estimate its non-reduced molecular weight as between 66 and $77 \mathrm{Kda}$. In earlier

drug disposition studies, autoradiography of electrophoresed plasma samples from rats administered ${ }^{14} \mathrm{C}$-labelled DP revealed that the radioactivity migrated to a position close to albumin. ${ }^{7314}$ An investigation in which ${ }^{35} \mathrm{~S}$-DL-penicillamine was administered to human volunteers showed by electrophoresis and autoradiography of plasma samples that the drug localised to a faint band at the trailing edge of albumin, ${ }^{17}$ that is, to a position similar to that which we now report for the DP conjugate detected by Western blotting. Our present studies throw some doubt on the conclusions of previous authors ${ }^{7} 1314$ that albumin is the target for DP binding. Firstly, although the DP conjugate migrated alongside the trailing edge of albumin it appeared as a horizontal and relatively narrow band whereas albumin (by staining with anti-albumin or amido black) was broad with a down-curved trailing edge in the position adjacent to the DP band. Second, in vitro incubations of purified human albumin with DP failed to generate the drug-derived conjugate at concentrations of the drug that readily generated conjugates in whole human plasma. We also considered the possibility that transferrin might be the target protein for DP binding. Although the DP band migrated at a very similar rate to transferrin on SDS-PAGE, it appeared slightly ahead of the transferrin; also, we did not demonstrate binding of DP when purified transferrin was incubated with the drug in vitro. However, the possibility that albumin or transferrin is the target protein for DP binding cannot be totally excluded since binding of DP may alter protein electrophoretic mobility, and factors in whole plasma may be required for conjugation of DP.

When we incubated fresh normal human plasma in vitro with concentrations of DP $(0.06-0.13 \mathrm{Mm})$ comparable to those reached in vivo in human patients ${ }^{18}$ a DP-derived antigen was detected by Western blot analysis that migrated to an identical position to that which was seen in plasma samples from patients receiving the drug. Since the DP conjugate generated in vivo can equally readily be generated in plasma in vitro, we conclude that there is no requirement for metabolic conversion of the drug by the tissues of the body (for example, the liver) to a reactive form. This is consistent with a simple spontaneous interaction of the drug (dimethylcysteine) with free protein sulphydryl groups to generate a disulphide-linked conjugate. At in vitro concentrations of DP $(0 \cdot 25-4 \mathrm{Mm})$ considerably higher than circulating levels reached in patients, further conjugates were detected, particularly two strong bands of higher molecular weight than the single band seen at low drug concentrations in vitro. We are unable to guess the identity of these proteins or speculate on their significance.

In conclusion, we have detected a single discrete DP-derived conjugate on a protein of molecular weight $66-77 \mathrm{Kda}$ in the plasma of patients taking DP as treatment for rheumatoid arthritis. Because of the limited number of patients in this study, and because the DP 
conjugate could not be quantified, we cannot at the present time make any conclusions about its significance with regard to either the pharmacological or toxicological actions of DP. Nevertheless, since DP is known to induce immunotoxic reactions in a large proportion of patients, many of which appear to be of a hypersensitivity type, it is reasonable to suggest that this conjugate may be the causative antigen in these reactions. Of course, to consolidate this hypothesis it would be necessary to demonstrate antibodies in patients' sera directed against a DP-derived antigen. It may also be of interest to examine whether drugs related to DP, such as aurothiomalate, might generate potential antigens in an analogous fashion. Further studies to identify the DP binding protein, and quantitation of the conjugate formed in different individuals may throw some light on the mechanism and predictability of DP toxicity.

This work was funded by the Arthritis and Rheumatism Council.

1 Hill HFH. Penicillamine in rheumatoid arthritis: adverse effects. Scand $\mathcal{F}$ Rheumatol Suppl 1979; 28: 94-99.

2 Emery P, Panayi G S. Autoimmune reactions to

D-penicillamine. In: Kammuller M E, Bloksma N, Seinen W, eds. Autoimmunity and toxicology: immune disregulation induced by drugs and chemicals. Amsterdam: Elsevier, 1987: 167-182.

3 Emery P, Panayi G S, Huston G, et al. D-penicillamineinduced toxicity in rheumatoid arthritis. The role of sulphoxidation status and HLA DR3. $\mathcal{F}$ Rheumatol 1984; 11: 626-32.

4 Coleman J W, Park B K. Antirheumatics: penicillamine. In:
Damani L A, ed. Sulphur containing drugs and related organic compounds. Chichester: Ellis Horwood, 1989; vol organic compounds.

5 Coleman J W. Allergic responses to drugs: current concepts and problems. Clin Exp Allergy 1990; 20: 79-85.

6 Amos H E. Diagnosis of allergic drug reactions. Int Archs Allergy 1973; 45: 218-31.

7 Planas-Bohne F. Metabolism and pharmacokinetics of penicillamine in rats - an overview. $\mathcal{f}$ Rheumatol Suppl 1981; 7: 35-40.

8 Coleman J W, Foster A L, Yeung J H K, Park B K. Drugprotein conjugates XV. A study of the disposition of $D$-penicillamine in the rat and its relationship to immunogenicity. Biochem Pharmacol 1988; 37: 737-42.

9 O'Donnell C A, Foster A L, Coleman J W. Penicillamine and penicillin can generate antigenic determinants on rat peritoneal cells in vitro. Immunology 1991; 72: 571-6.

peritoneal cells in vitro. Immunology 1991; 72: 571-6.
10 Storch W B. Clinical significance of penicillamine antibodies. Lancet 1988; ii: 214 .

11 Nagata N, Hurtenbach U, Gleichmann E. Specific sensitization of Lyt $-1^{+} 2^{-} \mathrm{T}$ cells to spleen cells modified by the drug D-penicillamine or a stereoisomer. F Immunol 1986; 136: 136-142.

12 O'Donnell C A, Coleman J W. A T-cell response to the antiarthritic drug penicillamine in the mouse: requirements for generation of the drug-derived antigen. Immunology 1992; 76: 604-9.

13 Joyce D A, Wade D N, Swanson B R. The pharmacokinetics of albumin conjugates of D-penicillamine in rats. Drug Metab Dispos 1989; 17: 208-211.

14 Joyce D A, Wade D N. Assay for D-penicillamine-protein conjugate in human plasma utilising chemical reduction followed by high-performance liquid chromatography with gold/mercury electrochemical detection. $\mathcal{f}$ Chromatog 1988; 430: 319-27.

15 Foster A L, Park B K, Coleman J W. A specific enzymelinked immunosorbent assay for definition of the IgE antibody response to disulphide-conjugated D-penicillamine in the rabbit. Int Archs Allergy 1987; 84: 271-6.

16 Laemmli U K. Cleavage of structural proteins during the assembly of the head of bacteriophage T4. Nature 1970; 227: $680-5$

17 Gibbs K, Walshe J M. Studies with ${ }^{35}$ S-labelled DLpenicillamine in patients with Wilson's disease. Quart $f$ Medicine 1971; XL: 275-87.

18 Butler M, Carruthers G, Harth M, et al. Pharmacokinetics of reduced D-penicillamine in patients with rheumatoid arthritis. Arth Rheum 1982; 25: 111-116. 